\title{
Trastornos mentales y consumo de drogas en la población víctima del conflicto armado en tres ciudades de Colombia
}

\author{
Guillermo Castaño1, Gloria Sierra1, Daniela Sánchez², Yolanda Torres', \\ Carolina Salas ${ }^{1}$, Carolina Buitrago ${ }^{1}$ \\ 1 Grupo de Investigación en Salud Mental, Universidad CES, Medellín, Colombia \\ 2 Grupo de Investigación en Psicología, Salud y Sociedad, Universidad CES, Medellín, Colombia
}

Introducción. La violencia en sus diferentes modalidades incrementa el riesgo de trastornos mentales y de consumo de drogas.

Objetivos. Estimar la prevalencia de los trastornos mentales, del uso y abuso de drogas, así como los factores asociados en víctimas de desplazamiento forzado en tres ciudades colombianas.

Materiales y métodos. Se hizo un estudio de prevalencia en una muestra de 1.026 personas entre los 13 y los 65 años de edad, a quienes se entrevistó utilizando el instrumento Composite International Diagnostic Interview y el Alcohol Use Disorders Identification Test de la Organización Mundial de la Salud, así como un cuestionario sobre el consumo de drogas modificado a partir de la encuesta del Sistema Interamericano de Datos Uniformes sobre Drogas de la Comisión Interamericana para el Control del Abuso de Drogas de la Organización de Estados Americanos, y otro sobre aspectos relacionados con el desplazamiento forzado. El análisis se hizo mediante el programa estadístico SPSSTM, versión 21.

Resultados. La prevalencia de vida de los trastornos mentales fue la siguiente: fobia específica, 17,7 \%; depresión mayor, 16,4\%; estrés postraumático, $9,9 \%$; trastorno oposicionista desafiante, $8,9 \%$; ansiedad por separación, 7,2\%; trastornos de conducta, 5,8\%, y déficit de atención, 5,6\%. La prevalencia de vida del consumo de alcohol fue de $68,7 \%$; de tabaco, 31,3\%, de marihuana, $11,2 \%$, de cocaína, 3,5 \%, de basuco, 2,0 \%, de inhalables, 2,3\%, y de medicamentos ansiolíticos sin receta, 2,5\%, en tanto que $0,7 \%$ de los entrevistados se había inyectado drogas. El presentar cualquiera de los trastornos mentales se asoció con el sexo femenino (odds ratio, $\mathrm{OR}=1,61 ; \mathrm{IC}_{95 \%} 1,21-2,14$ ), así como el haber sido sometido a más de un desplazamiento forzado $\left(\mathrm{OR}=1,47 ; \mathrm{IC}_{95} 1,05-2,05\right)$. El consumo de cualquiera de las drogas se asoció con ser hombre $\left(\mathrm{OR}=5,38 ; \mathrm{IC}_{95 \%}, 2,35-12,34\right)$.

Conclusiones. La alta prevalencia de trastornos mentales y de consumo de drogas en la población estudiada, comparada con la población general, constituye un problema de salud pública que justifica el diseño de planes y la implementación de programas orientados a la recuperación de su bienestar y salud mental.

Palabras clave: trastornos mentales; consumidores de drogas; violencia; Colombia.

https://doi.org/10.7705/biomedica.v38i0.3890

\section{Mental disorders and drug use in victims of the armed conflict in three cities in Colombia}

Introduction: Violence in its different forms increases the risk of mental disorders and the use of drugs. Objectives: To assess the prevalence of mental disorders, and the use and abuse of drugs, as well as associated factors in victims of forced displacement in three cities in Colombia.

Materials and methods: We conducted a prevalence study with 1.026 participants between 13 and 65 years old. Participants completed four surveys: the World Health Organization World Mental Composite International Diagnostic Interview, the World Health Organization Alcohol Use and Disorders Identification Test, a survey on drug use based on the Inter-American System of Uniform Drug-Use Data under the Inter-American Drug Abuse Commission of the Organization of American States, and a survey on aspects related to forced displacement. Data were analyzed using the SPSS ${ }^{\mathrm{TM}}$, version 21, software. Results: Lifetime prevalence of mental disorders was $17.7 \%$ for specific phobia, $16.4 \%$ for major depression, $9.9 \%$ for post-traumatic stress disorder, $8.9 \%$ for oppositional defiant disorder, $7.2 \%$ for separation anxiety disorder, $5.8 \%$ for conduct disorder, and $5.6 \%$ for attention deficit disorder. The lifetime prevalence of alcohol use was $68.7 \% ; 31,3 \%$ for tobacco; $11,2 \%$ for marihuana; $3.5 \%$ for cocaine; $2.0 \%$ for coca paste (basuco); $2.5 \%$ for non-prescription anxiolytic drugs, $2.3 \%$ for inhalants,

\section{Contribución de los autores:}

Guillermo Castaño: diseño del estudio

Gloria Sierra y Carolina Salas: procesamiento de datos

Daniela Sánchez y Carolina Buitrago: recolección de datos

Todos los autores participaron en el análisis de los datos y en la escritura del manuscrito. 
and $0.7 \%$ of participants had injected drugs. Presenting any mental disorder was associated with being female $(\mathrm{OR}=1,61$ IC95\%: 1,21-2,14) and experiencing more than one forced displacement (OR=1,47 IC95\%: 1,05-2,05). The use of any drug was associated with being male (OR=5,38 IC95\%: 2,35-12,34).

Conclusions: Compared to the general population, our study population exhibited high prevalence of mental disorders and drug use, emerging as a public health issue that calls for the design of plans and the implementation of programs aimed at recovering the mental health and well-being of this population.

Key words: Mental disorders; drug users; forced displacement; violence; Colombia. https://doi.org/10.7705/biomedica.v38i0.3890

Durante los conflictos armados existe un gran riesgo de que la salud mental individual y colectiva se vea afectada de forma inmediata, así como a mediano y largo plazo. La violencia en sus diferentes modalidades incrementa los riesgos de trauma psicológico; de hecho, los conflictos armados no solo generan muertes, heridas y discapacidades físicas, sino que también dejan huellas en la vida de las personas, las familias y la sociedad (1).

Asimismo, se producen cuantiosas pérdidas económicas y un aumento de la morbilidad psiquiátrica y de los problemas sociales derivados de los desplazamientos. La exposición a situaciones traumáticas puede producir un mayor nivel de angustia en las personas e, inclusive, afectar a amplios grupos de la comunidad que, aunque no sufran enfermedad mental alguna en el momento, sí experimentan reacciones de ansiedad y tristeza, así como alteraciones en el comportamiento (2).

En diversos estudios se han analizado los efectos más significativos tras un conflicto armado y el desplazamiento consecuente, entre ellos, el estrés postraumático, la ansiedad, la depresión, la ideación suicida, los ataques de pánico y el consumo de sustancias psicoactivas (3). Además, se ha establecido que la calidad de vida disminuye, se rompen las redes sociales y afectivas, se modifican los roles familiares y se produce desarraigo cultural (4).

En los estudios internacionales en los que se han analizado los efectos del conflicto armado en la salud mental de las víctimas, se han evidenciado prevalencias de vida de los síntomas del trastorno de estrés postraumático, la ansiedad y la depresión de $7,0,32,6$ y $22,2 \%$, respectivamente, y se ha señalado su asociación con la exposición al trauma subyacente (5). En las revisiones sistemáticas de

\footnotetext{
Correspondencia:

Guillermo A. Castaño, Grupo de Investigación en Salud Mental, Universidad CES, Calle 10 A N²2-04, Medellín, Colombia Teléfono: (574) 444 0525, extensión 1250; fax: (574) 2666046 gacastano@ces.edu.co
}

Recibido: 03/05/17; aceptado: 23/08/17 estudios realizados en personas expuestas a los conflictos armados y el desplazamiento en 40 países, se observaron prevalencias de 30,6\% para el trastorno de estrés postraumático y de $30,8 \%$ para el trastorno depresivo mayor (6).

La información sobre la prevalencia de los trastornos mentales y el consumo de drogas entre las víctimas del conflicto armado en Colombia, es escasa (7). En los estudios representativos hechos en el país entre población adulta víctima del conflicto armado, se ha registrado la presencia simultánea del trastorno de estrés postraumático, la ansiedad y la depresión (3). En su estudio, Londoño, et al., informaron de una prevalencia de síntomas de ansiedad entre 25,7 y $32,5 \%$, de trastorno de somatización entre 61 y $73,8 \%$, de abuso de alcohol de 23,8 a 38,1\%, y de trastornos de la alimentación entre 4,7 y $11,9 \%$ (8). También, se ha confirmado que un año después de la exposición a los hechos de victimización, los efectos psicológicos más frecuentes son el trastorno de estrés postraumático ( $37 \%)$, el trastorno de ansiedad generalizada ( $43 \%)$, la depresión mayor (38\%) y el riesgo de suicidio (45\%) (9).

El objetivo de este estudio fue dar cuenta de las prevalencias de los trastornos mentales y del consumo de drogas en población víctima del conflicto armado asentada en las tres ciudades colombianas que albergan el mayor número de víctimas de desplazamiento forzado del país: Medellín, Bogotá y Buenaventura, y estudiar las asociaciones estadísticas más relevantes.

\section{Materiales y métodos}

Se hizo un estudio observacional de corte transversal con un enfoque cuantitativo.

\section{Población y muestra}

Se estudiaron adultos y menores de edad incluidos en el Registro Único de Victimas del gobierno nacional sometidas a desplazamiento en los últimos diez años. Se excluyeron aquellas personas con síntomas de algún trastorno mental activo o con 
limitaciones cognitivas o neurolingüísticas que les impidiera comprender la entrevista, y quienes se negaron a firmar el consentimiento informado.

La población seleccionada incluyó a víctimas de desplazamiento forzado por la violencia que no estaban internadas en instituciones y residían en las tres ciudades que, según la Consultoría para los Derechos Humanos y el Desplazamiento (CODHES), recibieron la mayor cantidad de víctimas en el país en el 2013 (10): Medellín (13.664), Bogotá (25.506) y Buenaventura (19.792).

La encuesta se llevó a cabo en una muestra probabilística de 1.026 sujetos adolescentes y adultos entre los 13 y los 65 años de edad, calculada sobre el total de 58.962 personas reportadas como víctimas de desplazamiento forzado por el conflicto armado en las tres ciudades mencionadas, lo cual resultó en una muestra de 337 personas en Medellín, 347 en Bogotá y 342 en Buenaventura.

\section{Instrumento}

La información se recolectó con los siguientes instrumentos: la Entrevista Diagnóstica Internacional Compuesta (Composite International Diagnostic Interview, CIDI) de la Organización Mundial de la Salud (OMS); un cuestionario adaptado del Sistema Interamericano de Datos Uniformes sobre Consumo de Drogas (SIDUC) de la Comisión Interamericana para el Control del Abuso de Drogas de la Organización de Estados Americanos (CICAD/OEA), el cual incluyó los criterios de abuso o dependencia del Manual diagnóstico y estadístico de los trastornos mentales (Diagnostic and Statistical Manual of Mental Disorders, DSM IV), así como un cuestionario ad hoc sobre aspectos relacionados con el desplazamiento.

La CIDI es una entrevista muy estructurada diseñada para su uso por parte de entrevistadores debidamente entrenados. La entrevista permite generar diagnósticos psiquiátricos ajustados a las definiciones y los criterios de la CIE-10 y el DSMIV. La entrevista CIDI se adaptó y se validó para Colombia en el primer estudio nacional de salud mental realizado en el 2003 (10).

Además de la información para establecer los diagnósticos en el estudio, se incluyeron variables sociodemográficas.

\section{Procesamiento y análisis de la información}

Se hizo el análisis univariado de la información de los 1.026 sujetos de la muestra, y se calcularon las prevalencias de vida de los siguientes trastornos mentales: trastornos del estado del ánimo (depresión mayor, distimia, trastorno bipolar); trastornos de ansiedad (trastorno de pánico, agorafobia, ansiedad por separación, ansiedad generalizada, estrés postraumático, fobia específica y fobia social); y trastornos del impulso (trastorno negativista desafiante, trastorno de la conducta, déficit de atención).

Además, se usó el cuestionario ya mencionado para dar cuenta de las prevalencias de consumo de drogas con los criterios del DSM IV de abuso o dependencia de la marihuana, la cocaína y el basuco, en tanto que para los trastornos relacionados con el alcohol, se utilizó el AUDIT. La información sobre las variables relacionadas con el desplazamiento se recolectó mediante un cuestionario ad hoc.

Las variables cualitativas se expresaron mediante frecuencias absolutas y relativas. Los grupos se compararon mediante la prueba de independencia de ji al cuadrado o la prueba exacta de Fischer si procedía para las variables. La fuerza de asociación se estimó calculando las razones de disparidad (odds ratio, OR) con su respectivo intervalo de confianza $\left(\mathrm{IC}_{95 \%}\right)$.

La asociación entre variables asociadas al trastorno mental (incluidos los trastornos por uso de sustancias), se estableció calculando las OR crudas y sus intervalos de confianza de $95 \%$. Las asociaciones entre la exposición a eventos violentos relacionados con el desplazamiento, los trastornos mentales y el consumo de drogas, se consideraron significativas cuando el valor de $p$ era menor de 0,05. El análisis estadístico se hizo mediante el programa SPSSTM, versión 21.

\section{Consideraciones éticas}

Según los lineamientos de la Resolución 008430 de 1993 expedida por el Ministerio de Salud de Colombia, en la cual se establecen las normas científicas, técnicas y administrativas para la investigación en salud, el presente estudio se clasificó como de riesgo mínimo, pues no hubo ninguna intervención ni modificación intencionada de las variables biológicas, fisiológicas, psicológicas o sociales de los individuos que participaron en el estudio.

Se solicitó el consentimiento informado de los sujetos participantes y se obtuvo la aprobación del proyecto por parte del Comité de Ética de la Universidad CES. Asimismo, se protegió debidamente el anonimato y la privacidad de los participantes. Los procedimientos seguidos se acogieron a las 
normas éticas consignadas en la Declaración de Helsinki. Todos los participantes dieron su consentimiento informado por escrito antes de la entrevista, documento que reposa en poder del autor de correspondencia.

\section{Resultados}

\section{Características sociodemográficas y otras relacionadas con el desplazamiento}

Del total de personas encuestadas $(n=1.026)$, el $46,5 \%$ correspondía al sexo masculino y el $53,5 \%$ al femenino. Aproximadamente, una quinta parte eran menores de 18 años y 79,5 \% era población adulta; 91,1 \% pertenecía a estratos socioeconómicos bajos (estratos 1 y 2), y $85 \%$ tenía estudios de primaria o secundaria, en tanto que $8,8 \%$ había cursado estudios superiores.

En cuanto a las características relacionadas con el desplazamiento, el 80,8\% de los participantes habían sido desplazado una sola vez en su vida, los demás, dos o más veces; $85,6 \%$ de ellos habían sido desplazados con toda su familia; 5,8 \% habían sido desplazados menos de un año antes, y el $57,5 \%$, más de cinco años, pero menos de diez. Se encontró que frente a cada 100 personas desplazadas por los paramilitares, 173 habían sido desplazadas por acción de la guerrilla; en el 83,0 $\%$ de los casos, el desplazamiento había ocurrido de manera intempestiva y cerca de $69,1 \%$ de los desplazados fue acogido en la casa de un familiar (cuadro 1).

Los hechos de victimización que provocaron el desplazamiento fueron variados $y$, en muchas ocasiones, simultáneos, destacándose como los más frecuentes el despojo de tierras $(69,2 \%)$, las amenazas $(68,7 \%)$, los actos terroristas $(59,6 \%)$, el homicidio (27,5\%), y los delitos contra la libertad, las minas antipersona, el secuestro, la tortura o el involucramiento de menores en la guerra, en $10 \%$ de los casos o menos (figura 1).

\section{Trastornos mentales en la población desplazada por la violencia}

Los trastornos mentales de mayor prevalencia de vida en la población estudiada fueron: fobia específica (17,7 \%), depresión mayor (16,4\%), estrés postraumático $(9,9 \%)$, trastorno oposicionista desafiante $(8,9 \%)$, ansiedad por separación $(7,2 \%)$, trastornos de conducta $(5,8 \%)$, déficit de atención (5,6 \%), ansiedad generalizada (3,4 \%), agorafobia $(3,0 \%)$, trastorno de pánico $(2,8 \%)$, distimia y trastorno bipolar $(2,0 \%)$.
Las prevalencias de los trastornos mentales en el año anterior a la entrevista fueron las siguientes: fobia específica $(9,3 \%)$, estrés postraumático $(7,3 \%)$, depresión mayor $(7,1 \%)$, fobia social $(4,2 \%)$, ansiedad por separación $(3,2 \%)$, trastorno oposicionista desafiante $(2,2 \%)$, agorafobia $(2,0 \%)$, déficit de atención (2,1\%), distimia $(1,7 \%)$, ansiedad generalizada y trastorno de pánico $(1,4 \%)$, trastorno bipolar $(1,3 \%)$ y trastorno de conducta (1,2\%) (cuadro 2 ).

Un poco más de la quinta parte de los participantes $(21,25 \%)$ manifestó haber pensado en suicidarse alguna vez en su vida, 38,3\% de ellos había hecho un plan para consumar el suicidio, y $44,1 \%$ lo intentó al menos una vez.

Los trastornos mentales más prevalentes en el año anterior en cada ciudad fueron la fobia específica, la depresión mayor y el estrés postraumático, con la siguiente distribución: en Bogotá, fobia específica (10,37\%), depresión mayor (8,36 \%) y estrés postraumático (6,92 \%); en Medellín, fobia específica $(9,20 \%)$, depresión mayor $(8,01 \%)$ y

Cuadro 1. Variables relacionadas con el desplazamiento forzado de población víctima de violencia asentada en Bogotá, Medellín y Buenaventura, 2016

\begin{tabular}{|c|c|c|}
\hline Variable & $\mathbf{n}$ & $\%$ \\
\hline \multicolumn{3}{|l|}{ Número de desplazamientos } \\
\hline Uno & 829 & 80,8 \\
\hline Dos o más & 197 & 19,2 \\
\hline \multicolumn{3}{|l|}{ Forma de desplazamiento } \\
\hline Individual & 119 & 11,6 \\
\hline Familiar & 878 & 85,6 \\
\hline Grupal & 9 & $0, \mathrm{c}$ \\
\hline Masivo & 20 & $1, \mathrm{c}$ \\
\hline \multicolumn{3}{|l|}{$\begin{array}{l}\text { Tiempo transcurrido desde el } \\
\text { desplazamiento (años) }\end{array}$} \\
\hline Menos de uno & 59 & 5,8 \\
\hline Entre 1 y 5 & 377 & 36,7 \\
\hline Entre 6 y 10 & 590 & 57,5 \\
\hline \multicolumn{3}{|c|}{ Actor que influyó en el desplazamiento } \\
\hline Guerrilla & 596 & 58,1 \\
\hline Paramilitares & 345 & 33,6 \\
\hline Delincuencia común & 33 & 3,2 \\
\hline Ejército & 7 & 0,7 \\
\hline Otros & 45 & 4,4 \\
\hline \multicolumn{3}{|l|}{ Desplazamiento intempestivo } \\
\hline Sí & 893 & 87,0 \\
\hline No & 133 & 13,0 \\
\hline \multicolumn{3}{|c|}{ Lugar a donde llegó cuando fue desplazado } \\
\hline Casa de un familiar & 709 & 69,1 \\
\hline Institución gubernamental & 27 & 2,6 \\
\hline Albergues & 38 & 3,7 \\
\hline Terminal de transporte & 49 & 4,8 \\
\hline \multirow[t]{2}{*}{ Otro } & 203 & 19,8 \\
\hline & 1.026 & 100,0 \\
\hline
\end{tabular}




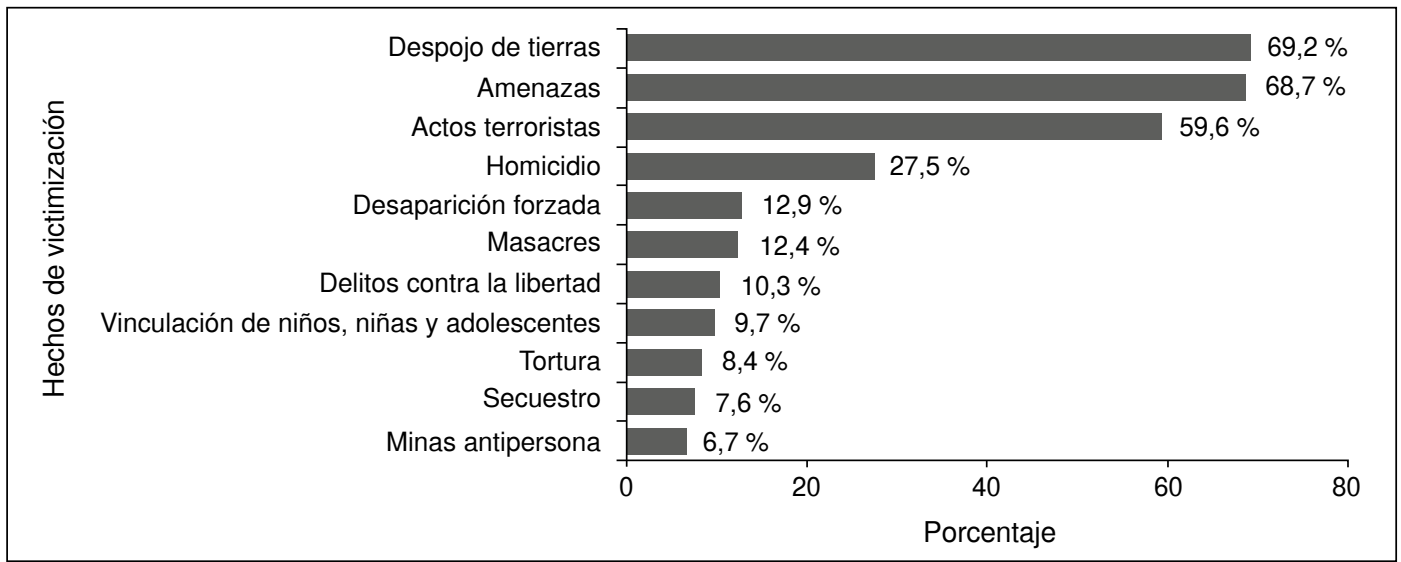

Figura 1. Distribución de los hechos de victimización en población sujeta a desplazamiento forzado asentada en Bogotá, Medellín y Buenaventura, 2016

Cuadro 2. Prevalencia de vida, en el año anterior y en el mes previo a la entrevista de los trastornos mentales en población víctima de desplazamiento forzado asentada en Medellín, Bogotá y Buenaventura, 2016 ( $n=1.026)$

\begin{tabular}{lccc}
\hline Trastorno & Prevalencia de vida & Prevalencia en el año anterior & Prevalencia en el mes previo \\
\cline { 2 - 4 } & $\left(\mathbf{I C}_{95 \%}\right)$ & $\left(\mathbf{I C}_{95 \%} \mathbf{)}\right.$ & $\mathbf{( I C}_{95 \%} \mathbf{)}$ \\
\hline Trastorno de pánico & $2,83(1,81-3,84)$ & $1,36(0,65-2,07)$ & $0,58(0,12-1,05)$ \\
Ansiedad generalizada & $3,41(2,30-4,52)$ & $1,36(0,65-2,07)$ & $0,39(0,01-0,77)$ \\
Agorafobia & $3,02(1,97-4,07)$ & $2,05(1,18-2,91)$ & $0,88(0,31-1,45)$ \\
Fobia social & $5,95(4,50-7,39)$ & $4,19(2,96-5,42)$ & $1,46(0,73-2,20)$ \\
Fobia específica & $17,74(15,40-20,08)$ & $9,26(7,49-11,03)$ & $2,73(1,73-3,73)$ \\
Estrés postraumático & $9,94(8,11-11,77)$ & $7,31(5,72-8,90)$ & $4,39(3,13-5,64)$ \\
Ansiedad por separación & $7,21(5,63-8,80)$ & $3,22(2,14-4,30)$ & $1,36(0,65-2,07)$ \\
Depresión mayor & $16,37(14,11-18,64)$ & $7,12(5,54-8,69)$ & $2,24(1,34-3,15)$ \\
Distimia & $2,05(1,18-2,91)$ & $1,66(0,88-2,44)$ & $0,78(0,24-1,32)$ \\
Trastorno bipolar & $2,05(1,18-2,91)$ & $1,27(0,58-1,95)$ & $0,88(0,31-1,45)$ \\
Déficit de atención & $5,62(3,83-7,40)$ & $2,03(0,94-3,12)$ & $\mathrm{NC}$ \\
Oposicionista desafiante & $8,89(6,69-11,10)$ & $2,18(1,05-3,32)$ & $\mathrm{NC}$ \\
Trastorno de conducta & $5,77(3,97-7,58)$ & $1,25(0,39-2,11)$ & $\mathrm{NC}$ \\
Bulimia & $0,63(0,00-1,49)$ & $0,31(0,00-0,92)$ & $0,31(0,00-0,92)$ \\
Anorexia & $0,31(0,00-0,92)$ & $0,00(0,00-0,00)$ & $0,00(0,00-0,00)$ \\
Cualquier trastorno mental & $42,88(39,86-45,91)$ & $26,22(23,53-28,91)$ & $11,89(9,91-13,87)$ \\
\hline
\end{tabular}

NC: no se calculó;

estrés postraumático $(6,53 \%)$, y en Buenaventura, estrés postraumático $(8,48 \%)$, fobia específica $(8,19 \%)$ y depresión mayor $(4,97 \%)$.

\section{Consumo, abuso y dependencia de drogas}

Los porcentajes de consumo de drogas a lo largo de la vida en la población de estudio fueron los siguientes: alcohol (68,7 \%), cigarrillo $(31,3 \%)$, marihuana $(11,2 \%)$, cocaína (3,5\%), basuco $(2,0 \%)$, substancias inhalables $(2,3 \%)$ y medicamentos ansiolíticos sin receta (2,5\%). En cuanto a otras drogas, se registró el consumo de LSD en $0,8 \%$ de los participantes, de ketamina en $0,2 \%$, de éxtasis en $0,5 \%$, del alucinógeno $2 \mathrm{CB}$ en $0,3 \%$, de anfetaminas en $0,1 \%$ y de otros alucinógenos en $0,97 \%$.

En el último año, la población objeto de estudio había consumido alcohol (46,5\%), tabaco (13,9\%), marihuana $(3,0 \%)$, cocaína $(1,2 \%)$, basuco $(0,5 \%)$, sustancias inhalables $(0,9 \%)$, medicamentos ansiolíticos sin receta $(1,7 \%)$, y otras drogas $(1,46 \%)$ como LSD $(0,2 \%)$, ketamina $(0,2 \%)$, éxtasis $(0,3 \%), 2 \mathrm{CB}(0,2 \%)$ anfetaminas $(0,1 \%)$ y otros alucinógenos $(0,5 \%)$, en tanto que el $0,7 \%$ reportó haberse inyectado drogas, siendo la heroína la droga elegida (cuadro 3). 
Con respecto a los trastornos por uso de sustancias en el último año, se encontró dependencia del alcohol en $0,8 \%$ y abuso en $1,7 \%$; dependencia $(1,4 \%)$ y abuso $(0,1 \%)$ de la marihuana; dependencia de la cocaína $(0,3 \%)$ y dependencia del basuco $(0,1 \%)$. No se reportó abuso de cocaína ni de basuco.

Por ciudades, los consumos más prevalentes de sustancias ilegales en el último año fueron los siguientes: en Bogotá, marihuana (3,46\%), medicamentos sin recetar que pueden producir dependencia (benzodiacepinas, derivados de opiáceos) $(2,59 \%)$ y cocaína (1,44\%); en Medellín: marihuana $(4,15 \%)$, medicamentos sin recetar $(1,78 \%)$, cocaína y sustancias inhaladas (1,48 \%), y en Buenaventura: marihuana $(1,46 \%)$, otras drogas (LSD, éxtasis, ketamina, sustancias inhaladas, hongos) $(1,17 \%)$, y medicamentos sin recetar y sustancias inhaladas $(0,88 \%)$.

\section{Variables asociadas con los trastornos mentales}

Variables sociodemográficas. En el análisis bivariado se encontró asociación con las variables 'sexo femenino' y 'adulto'; hubo diferencias en las proporciones de la ocupación: ser estudiante presentó asociación positiva con el hecho de presentar algún trastorno mental en el último año. Como factores asociados, el hecho de ser mujer presentó una OR de 1,61 (IC $\left.{ }_{95 \%} 1,21-2,14\right)$ comparado con ser hombre e, incluso más, con ser adulto (OR=2,18; IC $\left.{ }_{95 \%} 1,46-3,26\right)$, lo cual implica que se duplicó la probabilidad con respecto a ser adolescente, mientras que el hecho de ser estudiante resultó ser un factor de protección con relación a las personas que trabajaban, pues dicha probabilidad disminuyó en $40 \%$ (cuadro 4).
Variables relacionadas con el desplazamiento. Se encontró asociación con el número de desplazamientos $\left(O R=1,47 ; I_{95 \%} 1,05-2,05\right)$ en los casos de dos o más desplazamientos. También, se encontró asociación con el tiempo transcurrido desde el hecho de victimización: cuanto menor era dicho tiempo, mayor era el riesgo de presentar el trastorno mental en el último año, riesgo que disminuyó en más de $50 \%$ a medida que aumentó el tiempo transcurrido desde el momento del desplazamiento.

Todos los hechos de victimización, con excepción de la desaparición forzada y la forma del desplazamiento, se asociaron con el trastorno mental. Los hechos de victimización que presentaron mayor fuerza de asociación fueron los delitos contra la libertad $\left(O R=2,50 ; I_{95 \%} 1,66-3,78\right)$, las minas antipersona $\left(\mathrm{OR}=2,46 ; \mathrm{IC}_{95 \%} 1,50-4,05\right)$, y las masacres (OR=2,45; $\left.\mathrm{IC}_{95 \%} 1,67-3,59\right)$ (cuadro 5).

\section{Variables asociadas con los trastornos por uso de drogas}

Variables sociodemográficas. Se encontró asociación con la variable de sexo y se presentaron diferencias en las proporciones de la ocupación, siendo la de 'realizar quehaceres del hogar' la que presentó asociación positiva con el hecho de tener algún trastorno por uso de drogas. Como factor asociado, el hecho de ser hombre representó un riesgo 4,38 veces mayor que ser mujer, en tanto que el hecho de realizar labores en el hogar resultó ser un factor de protección comparado con trabajar, pues disminuyó en 77 \% la probabilidad de tener un trastorno por uso de drogas (cuadro 6).

Variables relacionadas con el desplazamiento. Las variables relacionadas con el desplazamiento y los trastornos por uso de drogas se asociaron

Cuadro 3. Prevalencia de vida, en el año anterior y en el mes previo a la entrevista del consumo de sustancias psicoactivas en población víctima de desplazamiento forzado asentada en Bogotá, Medellín y Buenaventura, 2016 ( $n=1.026)$

\begin{tabular}{|c|c|c|c|}
\hline \multirow[t]{2}{*}{ Sustancia psicoactiva } & \multirow[t]{2}{*}{$\begin{array}{l}\text { Prevalencia } \\
\text { de vida }\end{array}$} & \multirow{2}{*}{$\begin{array}{c}\text { Prevalencia en el año } \\
\text { anterior } \\
\left(I_{95 \%}\right)\end{array}$} & \multirow{2}{*}{$\begin{array}{c}\text { Prevalencia en el mes } \\
\text { previo }\end{array}$} \\
\hline & & & \\
\hline Alcohol & $68,71(65,88-71,55)$ & $46,49(43,44-49,54)$ & $24,46(21,83-27,09)$ \\
\hline Tabaco & $31,29(28,45-34,12)$ & $13,94(11,82-16,06)$ & $10,92(9,01-12,82)$ \\
\hline Marihuana & $11,21(9,28-13,14)$ & $3,02(1,97-4,07)$ & $2,05(1,18-2,91)$ \\
\hline Basuco & $2,05(1,18-2,91)$ & $0,49(0,06-0,91)$ & $0,10(0,00-0,29)$ \\
\hline Cocaína & $3,51(2,38-4,63)$ & $1,17(0,51-1,83)$ & $0,39(0,01-0,77)$ \\
\hline Alucinógenos & $0,97(0,37-1,58)$ & $0,49(0,06-0,91)$ & $0,00(0,00-0,00)$ \\
\hline Sustancias inhaladas & $2,34(1,41-3,26)$ & $0,88(0,31-1,45)$ & $0,39(0,01-0,77)$ \\
\hline Medicamentos ansiolíticos sin receta & $2,53(1,57-3,50)$ & $1,75(0,95-2,56)$ & $0,29(0,00-0,62)$ \\
\hline Sustancias inyectables & $0,68(0,18-1,19)$ & $0,39(0,01-0,77)$ & $0,00(0,00-0,00)$ \\
\hline Otras drogas & $1,46(0,73-2,20)$ & $0,78(0,24-1,32)$ & $0,00(0,00-0,00)$ \\
\hline
\end{tabular}


Cuadro 4. Factores asociados con los trastornos mentales según las características sociodemográficas de población víctima de desplazamiento forzado asentada en Medellín, Bogotá y Buenaventura

\begin{tabular}{|c|c|c|c|c|}
\hline \multirow[t]{2}{*}{ Variables } & \multicolumn{2}{|c|}{ Cualquier trastorno mental en el año anterior } & \multirow[t]{2}{*}{ OR (IC $\left.{ }_{95 \%}\right)$} & \multirow[t]{2}{*}{$\mathbf{p}$} \\
\hline & Sí (\%) & No (\%) & & \\
\hline \multicolumn{5}{|l|}{ Sexo } \\
\hline Hombre & 21,4 & 78,6 & 1 & 0,001 \\
\hline Mujer & 30,4 & 70,6 & $1,61(1,21-2,14)$ & \\
\hline \multicolumn{5}{|l|}{ Grupo etario } \\
\hline Adolescentes & 15,7 & 84,3 & 1 & 0,000 \\
\hline Adultos & 28,9 & 71,1 & $2,18(1,46-3,26)$ & \\
\hline \multicolumn{5}{|l|}{ Ocupación } \\
\hline Trabaja & 25,5 & 74,5 & 1 & 0,003 \\
\hline Estudia & 17,1 & 82,9 & $0,60(0,39-0,92)$ & \\
\hline No trabaja ni busca trabajo & 29,6 & 70,4 & $1,23(0,65-2,32)$ & \\
\hline Labores del hogar & 32,6 & 67,4 & $1,41(0,98-2,02)$ & \\
\hline Otra & 28,8 & 71,2 & $1,18(0,78-1,80)$ & \\
\hline \multicolumn{5}{|l|}{ Nivel socioeconómico } \\
\hline Sin estrato & 28,6 & 71,4 & 1 & 0,662 \\
\hline Bajo & 26,5 & 73,5 & $0,90(0,43-1,91)$ & \\
\hline Medio & 16,7 & 83,3 & $0,50(0,14-1,83)$ & \\
\hline No sabe & 21,9 & 78,1 & $0,70(0,23-2,13)$ & \\
\hline \multicolumn{5}{|l|}{ Régimen de seguridad social } \\
\hline Contributivo & 26,2 & 73,8 & 1 & 0,680 \\
\hline Subsidiado & 26,0 & 74,0 & $0,99(0,68-1,43)$ & \\
\hline No asegurado & 29,2 & 70,8 & $1,16(0,67-2,01)$ & \\
\hline Otro & 11,1 & 88,9 & $0,35(0,04-2,88)$ & \\
\hline \multicolumn{5}{|l|}{ Nivel de escolaridad } \\
\hline Sin estudios & 31,7 & 68,3 & 1 & 0,255 \\
\hline Primaria & 27,2 & 72,8 & $0,80(0,45-1,43)$ & \\
\hline Secundaria & 23,7 & 76,3 & $0,67(0,38-1,18)$ & \\
\hline Técnico/tecnólogo & 31,1 & 68,9 & $0,97(0,46-2,08)$ & \\
\hline Universitario & 36,7 & 63,3 & $1,24(0,50-3,10)$ & \\
\hline \multicolumn{5}{|l|}{ Estado civil } \\
\hline Soltero & 23,9 & 76,1 & 1 & 0,400 \\
\hline Casado & 32,2 & 67,8 & $1,51(0,92-2,49)$ & \\
\hline Unión libre & 26,8 & 73,2 & $1,17(0,86-1,60)$ & \\
\hline Separado/divorciado & 30,6 & 69,4 & $1,41(0,79-2,51)$ & \\
\hline Viudo & 31,8 & 68,2 & $1,49(0,59-3,74)$ & \\
\hline
\end{tabular}

con las amenazas $\left(\mathrm{OR}=4,02 ; \mathrm{IC}_{95 \%} 1,41-11,41\right)$, las masacres $\left(\mathrm{OR}=2,66 ; \mathrm{IC}_{95 \%} 1,26-5,61\right)$, y los homicidios (OR=2,21; $\left.\mathrm{IC}_{95 \%} 1,15-4,25\right)$ (cuadro 7).

\section{Discusión}

Durante las últimas cinco décadas, el conflicto armado en Colombia ha suscitado, entre otras crisis, la muerte de miles de ciudadanos vinculados o ajenos a él, así como el desplazamiento forzado de millones de personas. Pese a ello, son pocos los estudios hechos sobre la salud mental de las personas expuestas directamente a los distintos tipos de violencia relacionados con dicho conflicto (11).

Las cifras informadas por la Unidad para la Atención y Reparación Integral a las Víctimas señalan la existencia de 5'845.002 de víctimas hasta octubre del 2013. Entre los hechos a los que han estado expuestos las víctimas en el país, se encuentran los homicidios perpetrados contra las comunidades con el fin de forzar su desplazamiento, los actos de tortura, los tratos crueles, degradantes e inhumanos, el secuestro individual y colectivo como táctica de guerra, la desaparición forzada, las tomas armadas de diferentes poblaciones, las masacres indiscriminadas de civiles, el desplazamiento forzado masivo, los ataques, masacres y homicidios, los delitos contra la libertad, la integridad sexual y la violencia de género, las minas antipersona, el reclutamiento forzado de adultos y de menores, y el abandono o despojo forzado de tierras (12).

Dada la asociación estadística entre la presencia de factores de estrés psicosocial y el bienestar emocional, es de esperar que haya una alta prevalencia de síntomas o trastornos mentales entre las víctimas de conflictos armados $(13,14)$. 
Cuadro 5. Factores asociados a los trastornos mentales según las características del desplazamiento y de los hechos de victimización en población sujeta a desplazamiento forzado por la violencia asentada en Medellín, Bogotá y Buenaventura

\begin{tabular}{|c|c|c|c|c|}
\hline \multirow[t]{2}{*}{ Variables } & \multicolumn{2}{|c|}{ Cualquier trastorno mental en el año anterior } & \multirow[t]{2}{*}{ OR $\left(\mathrm{IC}_{95 \%}\right)$} & \multirow[t]{2}{*}{$\mathbf{p}$} \\
\hline & Sí (\%) & No (\%) & & \\
\hline \multicolumn{5}{|c|}{ Número de desplazamientos } \\
\hline Uno & 24,7 & 75,3 & 1 & 0,026 \\
\hline Dos o más & 32,5 & 67,5 & $1,47(1,05-2,05)$ & \\
\hline \multicolumn{5}{|c|}{ Forma de desplazamiento } \\
\hline Individual & 26,1 & 73,9 & 1 & 0,520 \\
\hline Familiar & 25,9 & 74,1 & $0,99(0,64-1,53)$ & \\
\hline Grupal & 33,3 & 66,7 & $1,42(0,33-6,02)$ & \\
\hline Masivo & 40,0 & 60,0 & $1,89(0,71-5,06)$ & \\
\hline \multicolumn{5}{|c|}{$\begin{array}{l}\text { Tiempo transcurrido desde el } \\
\text { desplazamiento (años) }\end{array}$} \\
\hline Menos de uno & 42,4 & 57,6 & 1 & 0,011 \\
\hline Entre 1 y 5 & 23,9 & 76,1 & $0,43(0,24-0,75)$ & \\
\hline Más de 5 & 26,1 & 73,9 & $0,48(0,28-0,83)$ & \\
\hline \multicolumn{5}{|l|}{ Actos terroristas } \\
\hline Sí & 29,5 & 70,5 & $1,53(1,14-2,05)$ & 0,004 \\
\hline No & 21,4 & 78,6 & 1 & \\
\hline \multicolumn{5}{|l|}{ Amenazas } \\
\hline Sí & 29,4 & 70,6 & $1,74(1,26-2,39)$ & 0,001 \\
\hline No & 19,3 & 80,7 & 1 & \\
\hline \multicolumn{5}{|c|}{ Delitos contra la libertad } \\
\hline Sí & 44,3 & 55,7 & $2,50(1,66-3,78)$ & 0,000 \\
\hline No & 24,1 & 75,9 & 1 & \\
\hline \multicolumn{5}{|c|}{ Desaparición forzada } \\
\hline Sí & 29,5 & 70,5 & $1,21(0,81-1,81)$ & 0,352 \\
\hline No & 25,7 & 74,3 & 1 & \\
\hline \multicolumn{5}{|l|}{ Homicidio } \\
\hline Sí & 37,9 & 62,1 & $2,20(1,63-2,96)$ & 0,000 \\
\hline No & 21,8 & 78,2 & 1 & \\
\hline \multicolumn{5}{|l|}{ Masacres } \\
\hline Sí & 43,3 & 56,7 & $2,45(1,67-3,59)$ & 0,000 \\
\hline No & 23,8 & 76,2 & 1 & \\
\hline \multicolumn{5}{|l|}{ Minas antipersona } \\
\hline Sí & 44,9 & 55,1 & $2,46(1,50-4,05)$ & 0,000 \\
\hline No & 24,9 & 75,1 & 1 & \\
\hline \multicolumn{5}{|l|}{ Secuestro } \\
\hline Sí & 37,2 & 62,8 & $1,75(1,08-2,83)$ & 0,022 \\
\hline No & 25,3 & 74,7 & 1 & \\
\hline \multicolumn{5}{|l|}{ Tortura } \\
\hline Sí & 41,9 & 58,1 & $2,18(1,39-3,44)$ & 0,001 \\
\hline No & 24,8 & 75,2 & 1 & \\
\hline \multicolumn{5}{|c|}{$\begin{array}{l}\text { Vinculación de niños, niñas y } \\
\text { adolescentes }\end{array}$} \\
\hline Sí & 39,0 & 61,0 & $1,93(1,26-2,97)$ & 0,002 \\
\hline No & 24,8 & 75,2 & 1 & \\
\hline \multicolumn{5}{|l|}{ Despojo de tierras } \\
\hline Sí & 30,8 & 69,2 & $2,37(1,69-3,34)$ & 0,000 \\
\hline No & 15,8 & 84,2 & 1 & \\
\hline
\end{tabular}

Hasta el 2012, los psicólogos de Médicos Sin Fronteras en Colombia habían atendido a 4.455 pacientes, de los cuales 3.286 eran mujeres (74\%) y 1.169 hombres ( $26 \%)$. En su mayoría, se trataba de mayores de 18 años (72\%), seguidos por el grupo de edad de 6 a 18 años $(25,5 \%)$ y, en tercer lugar, por los menores de 5 años (2,5\%).
Del análisis de los datos se desprende que gran parte de las personas que han acudido a la consulta clínica de esta organización no gubernamental ha vivido uno o más hechos relacionados con la violencia (67\%). Como consecuencia de ello y de otros factores de riesgo, se detectaron síntomas somáticos de origen psicológico 
Cuadro 6. Características sociodemográficas de la población de estudio desplazada por la violencia asentada en Medellín, Bogotá y Buenaventura y su relación con los trastornos por el uso de drogas, 2016

\begin{tabular}{|c|c|c|c|c|}
\hline \multirow[t]{2}{*}{ Variables } & \multicolumn{2}{|c|}{ Cualquier trastorno por uso de drogas } & \multirow[t]{2}{*}{ OR $\left(\mathrm{IC}_{95 \%}\right)$} & \multirow[t]{2}{*}{$\mathbf{p}$} \\
\hline & Sí (\%) & No (\%) & & \\
\hline \multicolumn{5}{|l|}{ Sexo } \\
\hline Hombre & 6,5 & 93,5 & $5,38(2,35-12,34)$ & 0,000 \\
\hline Mujer & 1,3 & 98,7 & 1 & \\
\hline \multicolumn{5}{|l|}{ Grupo etario } \\
\hline Adolescentes & 2,9 & 97,1 & $0,72(0,3-1,75)$ & 0,466 \\
\hline Adultos & 3,9 & 96,1 & 1 & \\
\hline \multicolumn{5}{|l|}{ Ocupación } \\
\hline Trabaja & 4,9 & 95,1 & 1 & 0,006 \\
\hline Estudia & 1,8 & 98,2 & $0,36(0,12-1,09)$ & \\
\hline No trabaja ni busca trabajo & 7,4 & 92,6 & $1,57(0,50-4,87)$ & \\
\hline Labores del hogar & 1,2 & 98,8 & $0,23(0,07-0,80)$ & \\
\hline Otra (pensionado, jubilado, etc.) & 6,7 & 93,3 & $1,42(0,64-3,12)$ & \\
\hline \multicolumn{5}{|l|}{ Estrato socioeconómico } \\
\hline Sin estrato & 5,7 & 94,3 & 1 & 0,456 \\
\hline Bajo & 3,9 & 96,1 & $0,66(0,15-2,86)$ & \\
\hline Medio & 0,0 & 100,0 & NC & \\
\hline No sabe & 0,0 & 100,0 & $\mathrm{NC}$ & \\
\hline \multicolumn{5}{|l|}{ Régimen de seguridad social } \\
\hline Contributivo & 4,9 & 95,1 & 1 & 0,454 \\
\hline Subsidiado & 3,3 & 96,7 & $0,65(0,30-1,42)$ & \\
\hline No asegurado & 4,2 & 95,8 & $0,84(0,25-2,80)$ & \\
\hline Otro & 11,1 & 88,9 & $2,42(0,27-21,47)$ & \\
\hline \multicolumn{5}{|l|}{ Nivel de escolaridad } \\
\hline Sin estudios & 1,6 & 98,4 & 1 & 0,112 \\
\hline Primaria & 2,5 & 97,5 & $1,62(0,20-13,03)$ & \\
\hline Secundaria & 4,4 & 95,6 & $2,87(0,38-21,66)$ & \\
\hline Técnico/tecnólogo & 8,2 & 91,8 & $5,54(0,63-48,83)$ & \\
\hline Universitario & 0,0 & 100,0 & NC & \\
\hline \multicolumn{5}{|l|}{ Estado civil } \\
\hline Soltero & 4,5 & 95,5 & 1 & 0,555 \\
\hline Casado & 2,3 & 97,7 & $0,50(0,11-2,15)$ & \\
\hline Unión libre & 3,0 & 97,0 & $0,65(0,31-1,35)$ & \\
\hline Separado/divorciado & 4,8 & 95,2 & $1,07(0,31-3,69)$ & \\
\hline Viudo & 0,0 & 100,0 & NC & \\
\hline
\end{tabular}

NC: no se calculó

relacionados con la depresión y la ansiedad, así como cuadros psicosomáticos y alteraciones del comportamiento (15).

Aunque hay estudios más recientes sobre la salud mental en la población general colombiana, los resultados del presente estudio se compararon con los datos del 2003 por su mayor similitud en la metodología utilizada. Los resultados de este estudio comparados con los obtenidos en la población general del país son un poco más altos, como se ha constatado en otros estudios. Según el Estudio Nacional de Salud Mental, 2003 (10), en la población entre los 18 y los 65 años de edad se informó una prevalencia de vida de cualquier trastorno mental de 40,1\%; en los 12 meses anteriores, dicha prevalencia fue de $16,6 \% \mathrm{y}$, en los 30 días previos, fue de $7,4 \%$, en tanto que las halladas en este estudio fueron mayores.
Las prevalencias informadas en los 12 meses anteriores al Estudio Nacional de Salud Mental del 2003 fueron las siguientes: depresión mayor, 5,6\%; trastorno bipolar, 0,8 \%; cualquier trastorno afectivo, 6,9 \%; fobia social, 2,5\%; trastorno de ansiedad generalizada, 0,7\%; trastorno de pánico, $0,6 \%$, y cualquier trastorno de ansiedad, $11,7 \%$ (10). En el presente estudio, dichas prevalencias fueron mayores: depresión mayor, 7,12 \%, trastorno bipolar, 1,27 \%, cualquier trastorno afectivo, $8,67 \%$, fobia social, $4,19 \%$, trastorno de ansiedad generalizada, $1,36 \%$, trastorno de pánico, 1,36 \% y cualquier trastorno de ansiedad, 20,8 \%.

Al comparar los hallazgos de esta investigación con los de otros estudios en población víctima de desplazamiento forzado, los resultados para algunos trastornos son similares y para otros varían, lo cual puede deberse a la metodología y a los 
Cuadro 7. Características del desplazamiento y de los hechos de victimización en población sujeta a desplazamiento forzado por la violencia asentada en Medellín, Bogotá y Buenaventura, y su relación con los trastornos por uso de drogas

\begin{tabular}{|c|c|c|c|c|}
\hline \multirow[t]{2}{*}{ Variables } & \multicolumn{2}{|c|}{ Cualquier trastorno por uso de drogas } & \multirow[t]{2}{*}{ OR $\left(\right.$ IC $\left._{95 \%}\right)$} & \multirow[t]{2}{*}{$\mathbf{p}$} \\
\hline & Sí (\%) & No (\%) & & \\
\hline \multicolumn{5}{|c|}{ Número de desplazamientos } \\
\hline Uno & 3,7 & 96,3 & $1,05(0,46-2,43)$ & 0,901 \\
\hline Dos o más & 3,6 & 96,4 & 1 & \\
\hline \multicolumn{5}{|c|}{ Forma de desplazamiento } \\
\hline Individual & 7,6 & 92,4 & 1 & 0,109 \\
\hline Familiar & 3,2 & 96,8 & $0,40(0,19-0,88)$ & \\
\hline Grupal & 0,0 & 100,0 & NC & \\
\hline Masivo & 5,0 & 95,0 & $0,64(0,08-5,37)$ & \\
\hline \multicolumn{5}{|c|}{$\begin{array}{l}\text { Tiempo transcurrido desde el } \\
\text { desplazamiento (años) }\end{array}$} \\
\hline Menos de uno & 6,8 & 93,2 & $2,00(0,68-5,82)$ & 0,198 \\
\hline Uno o más & 3,5 & 96,5 & 1 & \\
\hline \multicolumn{5}{|l|}{ Actos terroristas } \\
\hline Sí & 3,3 & 96,7 & $0,75(0,39-1,43)$ & 0,376 \\
\hline No & 4,3 & 95,7 & 1 & \\
\hline \multicolumn{5}{|l|}{ Amenazas } \\
\hline Sí & 4,8 & 95,2 & $4,02(1,41-11,41)$ & 0,005 \\
\hline No & 1,2 & 98,8 & 1 & \\
\hline \multicolumn{5}{|c|}{ Delitos contra la libertad } \\
\hline Sí & 5,7 & 94,3 & $1,67(0,68-4,08)$ & 0,260 \\
\hline No & 3,5 & 96,5 & 1 & \\
\hline \multicolumn{5}{|c|}{ Desaparición forzada } \\
\hline Sí & 5,3 & 94,7 & $1,56(0,67-3,62)$ & 0,297 \\
\hline No & 3,5 & 96,5 & 1 & \\
\hline \multicolumn{5}{|l|}{ Homicidio } \\
\hline Sí & 6,0 & 94,0 & $2,21(1,15-4,25)$ & 0,015 \\
\hline No & 2,8 & 97,2 & 1 & \\
\hline \multicolumn{5}{|l|}{ Masacres } \\
\hline Sí & 7,9 & 92,1 & $2,66(1,26-5,61)$ & 0,008 \\
\hline No & 3,1 & 96,9 & 1 & \\
\hline \multicolumn{5}{|c|}{ Minas antipersona } \\
\hline Sí & 5,8 & 94,2 & $1,67(0,58-4,85)$ & 0,340 \\
\hline No & 3,6 & 96,4 & 1 & \\
\hline \multicolumn{5}{|l|}{ Secuestro } \\
\hline Sí & 6,4 & 93,6 & $1,90(0,72-5,01)$ & 0,188 \\
\hline No & 3,5 & 96,5 & 1 & \\
\hline \multicolumn{5}{|l|}{ Tortura } \\
\hline Sí & 3,5 & 96,5 & $0,93(0,28-3,1)$ & 0,912 \\
\hline No & 3,7 & 96,3 & 1 & \\
\hline \multicolumn{5}{|c|}{$\begin{array}{l}\text { Vinculación de niños, niñas y } \\
\text { adolescentes }\end{array}$} \\
\hline Sí & 3,0 & 97,0 & $0,79(0,24-2,61)$ & 0,695 \\
\hline No & 3,8 & 96,2 & 1 & \\
\hline \multicolumn{5}{|c|}{ Despojo de tierras } \\
\hline Sí & 4,1 & 95,9 & $1,45(0,68-3,11)$ & 0,333 \\
\hline No & 2,8 & 97,2 & 1 & \\
\hline
\end{tabular}

instrumentos empleados. En este sentido, en una revisión sistemática que incluyó 20 estudios con personas refugiadas en países del este de Europa (13), se observó que el $9 \%$ de los participantes presentaba trastorno de estrés postraumático, comparado con $9,94 \%$ en el presente estudio; $5 \%$ presentaba trastorno depresivo mayor, comparado con $16,37 \%$ en el presente estudio, y $4 \%$, trastorno de ansiedad generalizada, comparado con $3,41 \%$ en este estudio.

En otra revisión del 2009, se resumieron los hallazgos de 161 estudios en los que participaron personas expuestas a conflictos armados y desplazamiento en 40 países, y las prevalencias fueron de 
$30,6 \%$ para el trastorno de estrés postraumático y de $30,8 \%$ para el trastorno depresivo mayor (6). Con respecto a estos dos trastornos, las prevalencias encontradas en este estudio fueron menores (9,94 y $16,37 \%$, respectivamente).

Al revisar los estudios realizados en Colombia en este tipo de población, los hallazgos también varían, lo cual puede explicarse por la sensibilidad y la especificidad del tipo de instrumentos utilizados. Algunos estudios son comparables con este por la utilización de los criterios diagnósticos del DSM IV para los trastornos mentales, entre ellos, el de Sánchez-Padilla, et al. (16), en 1.745 adultos de Ibagué y zonas rurales de Tolima, en el cual se informó una prevalencia de 21,9\% para el trastorno depresivo mayor comparada con una de $16,37 \%$ en este estudio; una prevalencia de $7,4 \%$ para el trastorno de estrés postraumático comparada con $9,94 \%$ en el presente estudio, y una de $32,9 \%$ para otros trastornos de ansiedad, porcentaje muy similar al hallado en la presente investigación (32,3\%).

En otros estudios, como el de Alejo, et al. (4), realizado en Bucaramanga en una muestra aleatoria estratificada de 851 adultos, se utilizó el protocolo de la Structured Clinical Interview for DSM-IV Axis I Disorders (SCID) y se encontró una prevalencia de $21 \%$ para el trastorno de estrés postraumático, menor a la hallada en nuestro estudio.

En el estudio de Echenique, et al. (17), en 142 adultos de Sincelejo, se utilizó la Clinician Rating Scale for Assessing Current and Lifetime PTSD, y se encontró una prevalencia de 45,2 \% para este trastorno, porcentaje superior al hallado en nuestro estudio.

En otros estudios consultados se usaron escalas distintas que imposibilitaban la comparación con los resultados de este; sin embargo, se incluyeron en las referencias, pues permiten apreciar diferentes estimaciones de las prevalencias de los trastornos mentales en este tipo de población. En el estudio de Andrade, et al. (18), se estimaron las siguientes prevalencias mediante el inventario de síntomas SCL-90-R de Derogatis en una muestra de 100 adultos entre 20 y 83 años de edad en Armenia: ideación paranoide, $14 \%$; depresión, $13 \%$; ansiedad fóbica, $12 \%$, y psicoticismo, $7 \%$.

Por su parte, Richards, et al. (19), utilizaron escalas basadas en el informe de los propios afectados sobre síntomas de ansiedad, síntomas depresivos y trastorno de estrés postraumático en 106 adultos de Medellín e informaron $88 \%$ de prevalencia para los síntomas de trastorno de estrés postraumático, 59 \% para los síntomas de ansiedad y $41 \%$ para los depresivos.

Por su lado, Sinisterra, et al. (20), utilizaron la Escala Estructurada Breve para el diagnóstico de trastorno de estrés postraumático en una población de 110 adultos entre 19 y 75 años de edad en Bogotá, e informaron una prevalencia de 97,3\% de posible trastorno de estrés postraumático.

En cuanto a los trastornos por consumo de drogas, se encontraron dos estudios que hacen referencia al consumo de alcohol en población víctima del conflicto armado: uno de Hewit-Ramírez, et al. (21), quienes aplicaron el Self Reporting Questionnaire y hallaron una prevalencia de síntomas de alcoholismo de $34 \%$, y otro estudio de Torres, et al., quienes reportaron 10,3\% (22). Todos estos porcentajes son superiores a los hallados en este estudio $(2,44 \%)$.

Con respecto a las prevalencias de consumo de drogas, según los hallazgos informados en el presente estudio que permiten su comparación con los de la población general colombiana (por haberse empleado la misma metodología e instrumento de medición), estas fueron menores para las drogas legales, como el alcohol y el cigarrillo, similares para sustancias como la marihuana, la cocaína y las drogas de síntesis, y mayores para el basuco y las sustancias inhaladas.

Según los datos del Estudio Nacional sobre el Consumo de Drogas del 2013 (23), el 42,1\% de las personas encuestadas declaró haber fumado tabaco alguna vez en la vida, y la tasa global de consumo en el momento de la encuesta o en el mes anterior fue de $12,9 \%$. Las prevalencias en la vida y del mes anterior halladas en este estudio en la población víctima de desplazamiento forzado, fueron menores $(31,99 \%$ y $10,92 \%$, respectivamente).

Por otro lado, en el estudio del 2013, el $87 \%$ de las personas declaró haber consumido alcohol al menos una vez en su vida. El consumo durante el año anterior fue de 58,8 \%, y el 35,8 \% declaró haber tomado alguna bebida alcohólica en los 30 días previos a la encuesta. Los porcentajes en nuestro estudio fueron menores (prevalencia en la vida, 68,71\%; prevalencia en el año anterior, 46,49 $\%$ y prevalencia en los 30 días previos, $24,26 \%$ ). Al analizar los trastornos por consumo de alcohol en el estudio en la población general, se encontró 
que $1,19 \%$ presentaba dependencia y 11,1\%, abuso en el consumo de alcohol. En este trabajo la prevalencia de la dependencia y el abuso fueron menores $(0,78 \%$ y $1,66 \%$, respectivamente).

En cuanto al consumo de marihuana, en el estudio en la población general del 2013 se reportó una prevalencia de vida de 11,5\%; en el año anterior, de $3,3 \%$ y en el mes previo, de 2,2 \%. En la población desplazada de este estudio dichas prevalencias fueron muy similares (prevalencia en la vida, $11,21 \%$, prevalencia en el año anterior, 3,02 \% y prevalencia en los 30 días previos, 2,05\%). En el estudio del 2013 , el $57,6 \%$ de los consumidores presentaba signos de abuso o dependencia en el año anterior. En el presente estudio, la cifra de trastornos por uso de esta sustancia (abuso y dependencia) fue de $1,5 \%$.

Las prevalencias de consumo de cocaína en la población víctima del desplazamiento forzado en este estudio también fueron muy similares a las encontradas en la población general colombiana, con excepción de la prevalencia de consumo del último año; 3,2 \% de la población entre los 12 y 65 años de edad en Colombia manifestó haber probado o usado esta sustancia alguna vez en su vida. El consumo en el año anterior fue de 0,7\%, y en el mes previo, de 0,4\%. Lo hallado en este estudio en población desplazada da cuenta de una prevalencia de vida de $3,51 \%$, en el año anterior de $1,17 \%$ y en los 30 días previos de 0,39\%. El porcentaje de personas que había abusado o tenía dependencia de esta sustancia en el estudio del 2013 fue de 0,42\%, en tanto que en el presente estudio fue mucho menor, de 0,29 \%.

Los datos sobre consumo de basuco en el país en el estudio del 2013 informan que 1,2 \% de la población lo había consumido alguna vez en su vida, 0,21 \% lo había hecho durante el año anterior y $0,17 \%$ en el mes anterior. En la población de este estudio, las cifras fueron mayores, con excepción de la prevalencia en el mes anterior, la cual fue menor (prevalencia de vida, 2,05\%; prevalencia en el año anterior, 0,49 \% y prevalencia en el mes previo, 0,10\%). En la población general colombiana, el $0,17 \%$ de los consumidores de basuco reunía los criterios de abuso o dependencia. En la población víctima de desplazamiento forzado de este estudio, la prevalencia de trastornos por uso de esta sustancia fue igual.

En cuanto a las sustancias inhaladas, según el estudio del 2013, 1,91\% de las personas encuestadas informó haber usado algún tipo de estas sustancias alguna vez en su vida, y 0,26 \% admitieron haberlo hecho en el año anterior. En la población víctima de desplazamiento forzado en este estudio, se encontraron prevalencias más altas (prevalencia de vida, 2,34 \% y en el año anterior, 0,88\%).

Con respecto a las denominadas drogas de síntesis (éxtasis, ketamina, 2CB, LSD), agrupadas como otras sustancias en la presente investigación dados los bajas porcentajes, la prevalencia de vida fue de $1,46 \%$, cifra cercana a lo encontrado en la población general del estudio del 2013 (1,64\%).

Por último, con respecto al consumo de drogas, es importante mencionar lo hallado en nuestro estudio con respecto al uso de drogas inyectadas, dadas las implicaciones en salud pública de las malas prácticas de inyección y su asociación con el HIV y los virus de las hepatitis B y C (24). La prevalencia de vida hallada en la población víctima del desplazamiento forzado da cuenta del 0,68 \% de personas que han incurrido en esta práctica y del 0,39 \% que lo hicieron en el año anterior a la encuesta. Debe señalarse que no se tienen datos sobre el consumo de drogas inyectadas en la población general colombiana.

Con respecto a las asociaciones con el trastorno mental, en este estudio se encontró que el hecho de ser mujer representó un riesgo por encima del $60 \%$ comparado con el de los hombres. Por otro lado, el hecho de ser adulto presentó una OR de 2,18 , lo que implica que se duplicó la probabilidad de tener un trastorno mental con respecto a los adolescentes. En este sentido, los datos de varios estudios epidemiológicos coinciden en mostrar que las mujeres tienen mayor prevalencia de trastornos del estado de ánimo, de ansiedad y psicóticos, en tanto que en los hombres son más frecuentes los trastornos por abuso de sustancias y el trastorno antisocial de personalidad (25-27); además, se ha encontrado una mayor prevalencia de trastornos mentales en el grupo etario entre los 25 y los 34 años (26).

En cuanto a la ocupación, se encontró que el ser estudiante era un factor de protección frente a los trastornos mentales, comparado con estar vinculado a un trabajo, con una probabilidad menor de padecerlos de $40 \%$. En este sentido, varios autores han encontrado una asociación importante, inversa e independiente entre el nivel educativo y la probabilidad de presentar trastornos mentales $(28,29)$. Por ejemplo, conforme se incrementa el nivel de escolaridad, disminuye la prevalencia 
del trastorno depresivo mayor; se ha encontrado que entre los individuos sin educación formal la prevalencia del trastorno depresivo mayor es de $8,0 \%$, y disminuye a $2,1 \%$ en aquellos con educación superior o de mayor nivel (30).

En cuanto a la asociación de las variables sociodemográficas, las relacionadas con el desplazamiento forzado, los hechos de victimización y su relación con los trastornos por consumo de drogas, se encontró que ser hombre representaba un riesgo 4,38 veces mayor para este trastorno que ser mujer, lo que coincide con los hallazgos de algunos autores que refieren que los trastornos por abuso de sustancias son más frecuentes en los hombres $(26,31)$. Con respecto a la ocupación, en el presente estudio se encontró que dedicarse a las labores del hogar protegía contra este tipo de trastorno, disminuyendo la probabilidad de presentarlo en el $77 \%$, aspecto que estaría relacionado con la ausencia de remuneración. Frente a ello, autores como $\mathrm{Wu}$, et al., consideran que tener ingresos económicos es un factor de riesgo, lo cual se suma al hecho de que la ocupación en las labores del hogar se asocia con el sexo, toda vez que son las mujeres las que más lo desempeñan (32).

Por otro lado, al analizar las variables relacionadas con el desplazamiento forzado y su asociación con los trastornos mentales y el consumo de drogas, es importante anotar que los estudios epidemiológicos y sociales han demostrado reiteradamente que el estrés psicosocial asociado con condiciones adversas de vida guarda relación con una mayor morbilidad y mortalidad por trastornos mentales y otras causas (33). Se sabe también que el estrés psicológico tiene un rol importante en el desencadenamiento y la evolución de los trastornos mentales, particularmente los trastornos por estrés postraumático (34), los trastornos por ansiedad y depresión (35), las psicosis esquizofrénicas y otras (36), y que constituye, a la vez, un factor de riesgo y un componente significativo en los trastornos por abuso y dependencia de sustancias (37).

La población objeto del presente estudio estuvo sometida a muchos hechos de victimización, pero las variables que resultaron asociadas con el trastorno mental fueron el haber sido sometida a más de un desplazamiento forzado $(\mathrm{OR}=1,47)$ y el tiempo transcurrido desde el hecho, observándose que, a menor tiempo, mayor riego de trastorno mental. Esto puede explicarse no solo por la intensidad de la exposición al evento, lo cual concuerda con lo señalado por varios autores en relación con el hecho de que el estrés ambiental y el psicológico cumplen un papel importante en el desencadenamiento y evolución de los trastornos mentales, sino también por la inmediatez del suceso $(34,38)$.

En este mismo sentido, los hechos de victimización que presentaron mayor asociación con el trastorno mental fueron los delitos contra la libertad, las minas antipersona y las masacres, eventos traumáticos que también han sido relacionados por otros autores con la presencia de un trastorno mental (39).

En cuanto a las limitaciones del estudio, es importante anotar que, aunque el diagnóstico de trastorno mental se estableció utilizando la entrevista clínica estructurada CIDI (OMS), lo que en principio da mayor confiabilidad al estudio, el diagnóstico de consumo de drogas se hizo con base en el informe de los propios participantes y no incluyó el análisis toxicológico, lo que no permite reflejar de manera exacta la existencia del problema en este grupo poblacional.

También cabe mencionar que se trató de un estudio retrospectivo en un grupo de personas víctimas del desplazamiento forzado asentado en las tres ciudades que albergan el mayor número de personas en esta condición y, por lo tanto, los resultados no se pueden generalizar a toda la población de víctimas en el país, pues la población estudiada presenta características y diferencias sociales y culturales significativas, no solo por su procedencia de diversas regiones, sino también por los contextos donde se asentaron, así como las diferencias étnicas, dado que estaba constituida básicamente por afrocolombianos.

En cuanto a los factores relacionados con el desplazamiento y los trastornos mentales, estos deben analizarse con cautela, pues no se hizo una regresión logística para valorar el efecto exacto de las variables independientes debido a que no constituía el objetivo principal del estudio.

Por último, es importante tener en cuenta que los resultados se compararon con los del Estudio de Salud Mental realizado en Colombia en el 2003 ante la imposibilidad de hacerlo con los del Estudio del 2015, pues en este se emplearon instrumentos y una metodología diferente (40).

Los resultados del estudio dan cuenta de prevalencias importantes de trastornos mentales en la población víctima del desplazamiento forzado en el 
marco del conflicto armado colombiano, superiores a las de la población general, por lo que es importante recordar que el diseño de planes y la implementación de programas orientados a la recuperación del bienestar de este colectivo son esenciales (41), y más cuando el Plan Decenal de Salud Pública, 2012-2021 (42) incluye la salud mental y la convivencia como una dimensión prioritaria.

En este sentido, el gobierno colombiano ha propuesto el Programa de Atención Psicosocial y Salud Integral a Víctimas (PAPSIVI) (43) como parte de las medidas de asistencia y rehabilitación contempladas en la Ley 1448 de 2011, la cual busca el restablecimiento de las condiciones físicas y psicosociales de la población víctima del conflicto armado en Colombia con base en la propuesta de atención primaria en salud de la OMS.

Al revisar la iniciativa se encuentran, sin embargo, algunos vacíos que han sido advertidos por la organización Médicos Sin Fronteras (15), los cuales deben superarse si se quiere dar una atención integral y oportuna en salud mental a la población desplazada por la violencia en Colombia. Entre dichos vacíos se destaca el que los equipos interdisciplinarios contemplados en el PAPSIVI no deben definirse únicamente en función de las víctimas reconocidas, ya que ello puede ser una barrera para el acceso a los servicios de salud mental en los sitios donde el conflicto ha tenido un impacto generalizado y no todos los afectados han alcanzado el reconocimiento oficial como víctimas.

Asimismo, debe mencionarse la necesidad de clarificar la responsabilidad y la composición de los equipos interdisciplinarios, y organizar su trabajo de forma coordinada con las instituciones y el personal responsable de la atención a las víctimas en las estructuras de salud del primer nivel de atención, así como el haber dejado en manos de las instituciones prestadoras de servicios de salud y de las entidades promotoras de salud la decisión sobre el tipo de atención que se ofrece en cada nivel, pues la única forma de garantizar una atención de calidad a las víctimas del conflicto es delimitando las diferentes responsabilidades en el ámbito de la promoción, la prevención, el tratamiento y la rehabilitación, y facilitando el acceso oportuno y la continuidad a los servicios, sobre todo en las zonas rurales, de manera que la población no tenga que desplazarse a centros hospitalarios de segundo nivel en las capitales departamentales.

\section{Conflicto de intereses}

Los autores declaran no tener conflicto de intereses.

\section{Financiación}

El estudio fue financiado por Colciencias, convocatoria 711/2015, contrato RC No. 841 de 2015.

\section{Referencias}

1. Rodríguez J, De La Torre A, Miranda C. La salud mental en situaciones de conflicto armado. Biomédica. 2002;22:33746. https://doi.org/10.7705/biomedica.v22iSupp2.1183

2. World Health Organization. Mental health of refugees. Genève: World Health Organization; 1996. Fecha de consulta: 15 de junio de 2017. Disponible en: http://apps. who.int/disasters/repo/8699.pdf

3. Bell V, Méndez F, Martínez C, Palma PP, Bosch M. Characteristics of the Colombian armed conflict and the mental health of civilians living in active conflict zones. Confl Health. 2012;6:10. https://doi.org/10.1186/1752-1505-6-10

4. Alejo E, Rueda G, Ortega M, Orozco C. Estudio epidemiológico del trastorno por estrés postraumático en población desplazada por la violencia política en Colombia. Univ Psychol. 2007;6:623-35.

5. Husain F, Mark A, López B, Becknell K, Blanton C, Araki $D$, et al. Prevalence of war-related mental health conditions and association with displacement status in postwar Jaffna district, Sri Lanka. JAMA. 2011;306:522-5. https://doi.org/10. 1001/jama.2011.1052

6. Steel Z, Chey T, Silove D, Marnane C, Bryant A, van Ommeren M. Association of torture and other potentially traumatic events with mental health outcomes among populations exposed to mass conflict and displacement: A systematic review and meta-analysis. JAMA. 2009;302: 537-49. https://doi.org/10.1001/jama.2009.1132

7. Campo A, Oviedo H, Herazo E. Prevalencia de síntomas, posibles casos y trastornos mentales en víctimas del conflicto armado interno en situación de desplazamiento en Colombia: una revisión sistemática. Rev Colomb Psiquiatr. 2014;43:177-85. https://doi.org/10.1016/j.rcp.2014.07.003

8. Londoño A, Romero P, Casas G. The association between armed conflict, violence, and mental health: A cross sectional study comparing two populations in Cundinamarca department, Colombia. Confl Health. 2012;6:1-6. https://doi. org/10.1186/1752-1505-6-12

9. Londoño NH, Muñoz O, Correa JE, Patiño C, Jaramillo G, Raigoza J, et al. Salud mental en víctimas de la violencia armada en Bojayá (Chocó, Colombia). Rev Colomb Psiquiatr. 2005;34:493-505.

10. Ministerio de la Protección Social. Estudio Nacional de Salud Mental Colombia, 2003. Fecha de consulta: 23 de mayo de 2017. Disponible en: https://www.minsalud.gov. co/Documentos\%20y\%20Publicaciones/ESTUDIO\%20 NACIONAL\%20DE\%20SALUD\%20MENTAL\%20EN\%20 COLOMBIA.pdf

11. Imbusch $\mathbf{P}$, Misse $\mathbf{M}$, Carrión $\mathbf{F}$. Violence research in Latin America and the Caribbean: A literature review. Int $\mathrm{J}$ Conflict Violence. 2011;5:87-154. https://doi.org/10.4119/UNIBI/ijcv.141

12. Unidad para la Atención y Reparación Integral a las Víctimas. Índice de riesgo de victimización. 2012. Fecha de consulta: 8 mayo de 2017. Disponible en: http://rni. unidadvictimas.gov.co/IRV 
13. Fazel M, Wheeler J, Danesh J. Prevalence of serious mental disorder in 7000 refugees resettled in western countries: A systematic review. Lancet. 2005;365:1309-14. https://doi.org/10.1016/S0140-6736(05)61027-6

14. Badri A, Crutzen R, van den Borne HW. Exposures to war-related traumatic events and post-traumatic stress disorder symptoms among displaced Darfuri female university students: An exploratory study. BMC Public Health. 2012;12:603. https://doi.org/10.1186/1471-2458-12-603

15. Médicos sin Fronteras. Las heridas menos visibles: salud mental, violencia y conflicto armado en el sur de Colombia. 2013. Fecha de consulta: 25 de mayo de 2016. Disponible en: https://www.msf.es/sites/default/files/legacy/adjuntos/ Informe-Colombia_Junio-2013.pdf

16. Sánchez-Padilla E, Casas G, Grais RF, Hustache S, Moro MR. The Colombian conflict: A description of mental health program in the Department of Tolima. Confl Health. 2009;3:13. https://doi.org/10.1186/1752-1505-3-13

17. Echenique C, Medina LM, Medina AR, Ramírez A. Prevalencia del trastorno por estrés postraumático en población desplazada por violencia en Sincelejo. Psicol Caribe. 2008; 21:122-35.

18. Andrade JA, Parra M, Torres LD. Tendencias psicopatológicas en personas desplazadas y reasentadas en personas desplazadas en el departamento del Quindío entre 2005 y 2011. Agora USB. 2011;11:279-96. https://doi.org/10.21500/ 16578031.395

19. Richards A, Ospina J, Barrera M, Escobar J, Ardila M, Metzler T. Posttraumatic stress disorder, anxiety and depression symptoms, and psychosocial treatment needs in Colombians internally displaced by armed conflict: A mixed method evaluation. Psychol Trauma. 2011;3:384-93. https:// doi.org/10.1037/a0022257

20. Sinisterra M, Figueroa FS, Moreno VF, Robayo MF, Sanguino JF. Prevalencia del trastorno de estrés postraumático en población en situación de desplazamiento en la localidad de Ciudad Bolívar, Bogotá, Colombia, 2007. Psychologia. 2010;4:83-97.

21. Hewitt N, Juárez F, Parada AJ, Guerrero J, Romero Y, Salgado AM, et al. Afectaciones psicológicas, estrategias de afrontamiento y niveles de resiliencia de adultos expuestos al conflicto armado en Colombia. Rev Colomb Psiquiatr. 2016;25:125-40. https://doi.org/10.15446/rcp.v25n1.49966

22. Torres Y, Bareno J, Sierra GM, Mejía R, Berbesi DY. Indicadores de situación de riesgo de salud mental población desplazada Colombia. Rev Observ Nac Salud Mental. 2012;1:28-38.

23. Ministerio de Justicia y del Derecho, Observatorio de Drogas en Colombia, Ministerio de Salud y Protección Social. Estudio Nacional de Consumo de Sustancias Psicoactivas en Colombia, 2013. Fecha de consulta: 3 de mayo 2017. Disponible en: http://www.descentralizadrogas. gov.co///pdfs/documentacion/estudios/Estudio_Consumo_ SPA_Colombia_2013_UNODC.pdf

24. De la Fuente L, Barrio G, Vicente J, Bravo MJ, Santacreu J. The impact of drug-related deaths on mortality among young adults in Madrid. Am J Public Health. 1995;85:102-5.

25. Canino GJ, Bird HR, Shround PE, Rubio M, Bravo M, Martínez R. Prevalence of specific psychiatric disorders in Puerto Rico. Arch Gen Psychiatry. 1987;44:727-35. https:// doi.org/10.1001/archpsyc.1987.01800200053008
26. Kessler RC, McGonagle KA, Zhao S, Nelson CB, Hughes M, Eshleman S. Lifetime and 12 - month prevalence of DSM-III-R psychiatric disorders in the United States results from the National Comorbidity Survey. Arch Gen Psychiatry. 1994;51:8-19. https://doi.org/10.1001/ archpsyc.1994.03950010008002

27. Patel V, Araya R, de Lima M, Ludermir A, Todd C. Women poverty and common mental disorders in four restructuring societies. Soc Sci Med. 1999;49:1461-71. https://doi.org/10. 1016/S0277-9536(99)00208-7

28. Posada JA, Trevisi C. Prevalencia, severidad y necesidades no satisfechas del tratamiento de los trastornos de ansiedad, relacionados con sustancias, del estado del ánimo y del control de los impulsos en adultos según el Estudio Nacional de Salud Mental, Colombia, 2003. Med Unab. 2004;7:65-72.

29. Noorbala AA, Bagheri Yazdi SA, Yasamy MT, Mohammad K. Mental Health Survey of the adult population in Iran. Br J Psychiatry. 2004;184:70-3. https://doi.org/10.1192/ bjp.184.1.70

30. Belló M, Puentes-Rosas E, Medina ME, Lozano R. Prevalence and diagnosis of depression in México. Salud Pública Mex. 2005;47:S4-11.

31. Bello LM, Rodríguez N, Trujillo S, Serrano J. Consumo de alcohol en Canarias: tendencia y factores sociodemográficos asociados más importantes (1997-2009). Rev Esp Nutr Comunitaria. 2014;20:57-6. https://doi.org/10.7400/ RENC.2014.01.2.5012

32. Wu L, Schlenger WE, Galvin DM. The relationship between employment and substance use among students aged 12 to 17. J Adolescent Health. 2003;32:5-15. https:// doi.org/10.1016/S1054-139X(02)00447-0

33. McFarlane AC. Traumatic stress in the 21st century. Aust N Z J Psychiatry. 2000;34:896-902. https://doi.org/10.1080/ 000486700264

34. Paykel ES. Stress and affective disorders in humans. Semin Clin Neuropsychiatry. 2001;6:4-11.

35. Mumford DB, Minhas FA, Akhtar I, Akhter S, Mubbashar MH. Stress and psychiatric disorder in urban Rawalpindi. Community survey. Br J Psychiatry. 2000;177:557-62. https://doi.org/10.1192/bjp.177.6.557

36. Yui K, Goto K, Ikemoto S, Ishiguro T. Stress induced spontaneous recurrence of methamphetamine psychosis: The relation between stressful experiences and sensitivity to stress. Drug Alcohol Depend. 2000;58:67-75. https://doi. org/10.1016/S0376-8716 (99)00060-5

37. Gómez MB, Primm AB, Tzolova-lontchev I, Perry W, Vu HT, Crum RM. A description of precipitants of drug use among dually diagnosed patients with chronic mental illness. Community Ment Health J. 2000;36:351-62.

38. Agid O, Kohn Y, Lerer B. Environmental stress and psychiatric illness. Biomed Pharmacother. 2000;54:135-41. https://doi.org/10.1016/S0753-3322(00)89046-0

39. McDonald L. Psychosocial rehabilitation of civilians in conflict-affected settings. En: Erin Martz, editor. Trauma rehabilitation after war and conflict, community and individual perspec-tives. USA: Springer; 2010. p. 215-45.

40. Ministerio de la Protección Social. Estudio Nacional de Salud Mental Colombia, 2015. Fecha de consulta: 12 
de mayo de 2017. Disponible en: https://www.odc.gov.co/ Portals/1/publicaciones/pdf/consumo/estudios/nacionales/ CO031102015-salud_mental_tomol.pdf

41. Kohn R, Levav I, Caldas JM, Vicente B, Andrade L, Caraveo-Anduaga JJ. Los trastornos mentales en América Latina y el Caribe: asunto prioritario para la salud pública. Rev Panam Salud Pública. 2005;18:229-40. https://doi. org/10.1590/S1020-49892005000900002

42. Ministerio de Salud y Protección Social. Plan decenal de salud pública 2012-2021. Bogotá, D. C., 2013. Fecha de consulta: 28 de abril de 2017. Disponible en: https://www. minsalud.gov.co/Documentos\%20y\%20Publicaciones/ Plan\%20Decenal\%20-\%20Documento\%20en\%20 consulta\%20para\%20aprobaci\%C3\%B3n.pdf

43. Ministerio de Salud y Protección Social. Programa de atención psicosocial y salud integral a víctimas, (PAPSIVI). Bogotá: Ministerio de Salud y Protección Social; 2013. Fecha de consulta: 3 de mayo de 2017. Disponible en: http://www.minsalud.gov.co/proteccionsocial/Paginas/ Victimas PAPSIVI.aspx 